\section{Effects of Adjuvant Oxaliplatin on Anal Function in Locally Advanced Rectal Cancer Treated with Preoperative Chemo- Radiotherapy and Low Anterior Resection}

\section{Abstract}

Background: The main objective of this study was to find out if the application of adjuvant oxaliplatin (OXA) influences sphincter function in patients with locally advanced rectal cancer (LARC) treated with preoperative capecitabine/ radiotherapy followed by lower anterior resection (LAR).

Methods: Patients with LARC treated at our medical center with LAR and with absence of loco-regional relapse for at least two years were retrospectively analyzed independently of the type of adjuvant treatment that they received. Anal sphincter function was assessed by Wexner's incontinence score (0 to 20 points, with score points inversely proportional to sphincter function). All questionnaires were completed between January 2010 and December 2012.

Sphincter function as assessed by the Wexner test was compared between patients with and without OX treatment, using the non-parametrical Mann-Whitney test. Statistical analyses were conducted with IBM SPSS Statistics package, version 20.

Results: 92 patients were included in our study since 2006. The mean time from LAR to fecal function assessment was 58 months (25 to 96 months). Wexner test median values did not differ significantly $(p=0.450)$ between patients with and without adjuvant OXA. The median (IQR) for the group of patients without adjuvant OXA treatment was 6.0 (1.0-11.25), and of 5.0 (1.0-10.0) for the group that received adjuvant OXA.

Conclusions: Based on our findings, OXA could be used as adjuvant treatment in LARC according to the absence of deleterious effects over sphincter anal function. Due to the retrospective nature of our study, prospective studies should be warranted in similar scenarios.

Keywords: Oxaliplatin; Rectal cancer; Anal function; Radiotherapy

Received: February 12, 2017; Accepted: February 20, 2017; Published: February 27, 2017

\section{Introduction}

It is estimated that in 2015 more than 132000 cases of colorectal cancer (CRC), of which 30 percent arise in the rectum [1], will be diagnosed in the United States with about 50000 deaths in the same period [2].

Particularly for locally advanced rectal adenocarcinoma (LARC), the risk of local recurrence rates in rectal cancer has declined from $40 \%$ to less than $10 \%$, with significant influence in 5 -year-survival
Fernando Arias ${ }^{1}$, Antonio Viudez², Clara Eito ${ }^{1}$, Berta Ibanez-Beroiz³, Gemma Asin ${ }^{1}$, Irene Hernandez², Koldo Cambra ${ }^{3}$, Marta Errasti ${ }^{1}$, Marta Barrado', Maider Campo', Ignacio Visus ${ }^{1}$, Sonia Flamarique ${ }^{2}$ and Miguel A Ciga ${ }^{4}$

1 Radiation Oncology Department, Complejo Hospitalario de Navarra, Pamplona, Spain

2 Medical Oncology Department, Complejo Hospitalario de Navarra, Pamplona, Spain

3 Methodology and Statistics Department, Complejo Hospitalario de Navarra, Pamplona, Spain

4 Surgery Department, Complejo Hospitalario de Navarra, Pamplona, Spain

Corresponding author: Fernando Arias

झ farias.delavega@outlook.es

Radiation Oncology Department, Complejo Hospitalario de Navarra, Irunlarrea 3, Pamplona, 31008, Spain.

Tel: 0034848422874

Fax: 0034848423303

Citation: Arias F, Viudez A, Eito C, et al. Effects of Adjuvant Oxaliplatin on Anal Function in Locally Advanced Rectal Cancer Treated with Preoperative ChemoRadiotherapy and Low Anterior Resection. Colorec Cancer 2017, 3:1. 
rates up to $71 \%$. This is a consequence of surgical approaches by total mesorectal excision (TME) [3]. Consequently, TME is considered one of the most influential factors in rectal cancer outcomes and a key part of clinical practice [4].

Nowadays, neoadjuvant therapy based on fluoropirimidine chemotherapy in concurrent combination with long-course radiation (ChRT) is regarded as the standard care for LARC. This confers better local control rates than surgery alone, although it does not offer a clear increase in survival [5].

Oxaliplatin (OXA) is a third generation platinum drug with a reduced cross-reactivity with DNA. Nevertheless, OXA possess capabilities of permanently damaging DNA by evading repair systems [6] This chemotherapy agent is routinely used as an adjuvant in metastatic colorectal cancer $[7,8]$. However, the use of OXA specifically for LARC focuses in the adjuvant setting although its efficacy in this context is still controversial [9]. OXA-induced neuropathy is the most prominent adverse effect, both during and after completion of chemotherapy. This kind of neurotoxicity is usually dose-limiting (cumulative doses $>540 \mathrm{mg}$ / $\mathrm{m}^{2}$ ) and shows long-lasting adverse events that may jeopardize therapeutic outcome and the quality-of-life of CRC patients [10]. Knowledge on the pathophysiology and clinical profile of this kind of sequela is being increased. Two types of neuropathy are usually observed: acute neuropathy with transient symptoms that occur shortly after administration. This is characterized by paresthesias and dysaesthesias of hands, feets and peroral region with jaw tightness, and also chronic neuropathy characterized by distal pareshesia, sensory ataxia, functional impairment, jaw and eye pain, ptosis, leg cramps and visual and voice changes $[11,12]$. Recently published evidence suggests a link between the acute symptoms and the development of chronic NTX.

The effect of OXA on neuro vegetative function has not yet been sufficiently investigated. We hypothesized that adjuvant OXAbased treatment could affect the anal function of patients with LARC who received preoperative radiation followed by lower anterior resection (LAR).

The main objective of this study is to find out if there is a correlation between adjuvant OXA-based treatment and sphincter function (SF) in patients with LARC previously treated with homogeneous preoperative capecitabine/radiotherapy followed by LAR with (TME).

\section{Materials and Methods}

Patients diagnosed with LARC began to be treated from 2004 with preoperative chemoradiation based on capecitabine (825 $\mathrm{mg} / \mathrm{m}^{2}$ day and $50.4 \mathrm{~Gy}$ at $1.8 \mathrm{~Gy}$ per fraction) at our medical center (Complejo Hospitalario de Navarra, Pamplona, Navarre, Spain). Six to eight weeeks after CTRT, all patients were operated by TME. Most patients received postoperative chemotherapy according to tumor response, clinical stage and hypothetical risk of relapse.

The Institutional Review Board (IRB) of Navarre (Spain) approved this study, in which we retrospectively reviewed 92 consecutive patients from 2006 with LARC treated at our center with LAR approach, and with more than 2 years without local and/or distant relapse. Ninety-five per cent of them authorized their participation in our study.

Fecal incontinence was measured using the validated Wexner incontinence score (from 0 to 20) [13]. This scale has become widely used for the assessment of severity of fecal incontinence, as it is straightforward and easy to understand by patients. A higher Wexner score indicates more symptoms of fecal incontinence. All questionnaires were filled from January 2010 to December 2012. Pathological response was measured using the Mandard criteria [14].

\section{Statistical analysis}

Sample characteristics were summarized using average and standard deviations, median and interquartile ranges (IQR), frequency and percentages, depending on the nature of the variables. Sphincter function as assessed by the Wexner test in patients with and without adjuvant OXA was compared with the Mann-Whitney test, and graphically represented by box-plots. Statistical analyses were performed with the IBM SPSS Statistics package, version 20 .

\section{Results}

Patient characteristics are shown in Table 1.60 out of the 92 patients were males (65\%). $50 \%$ of the cases were located in the medium rectum $(5-10 \mathrm{~cm}$ from anal margin). Including the data from all patients with LARC treated at our center, the rate of sphincter preservation was $75 \%$. Most of these cases were cT3 stage (90\%), $78 \%$ of them with nodal involvement. Thirty nine patients (42.4\%) had Tumor Regression Grade (Mandard) Grade 1-2; 74/92 patients (81\%) received 6 cycles of adjuvant

Table 1 Patient characteristics.

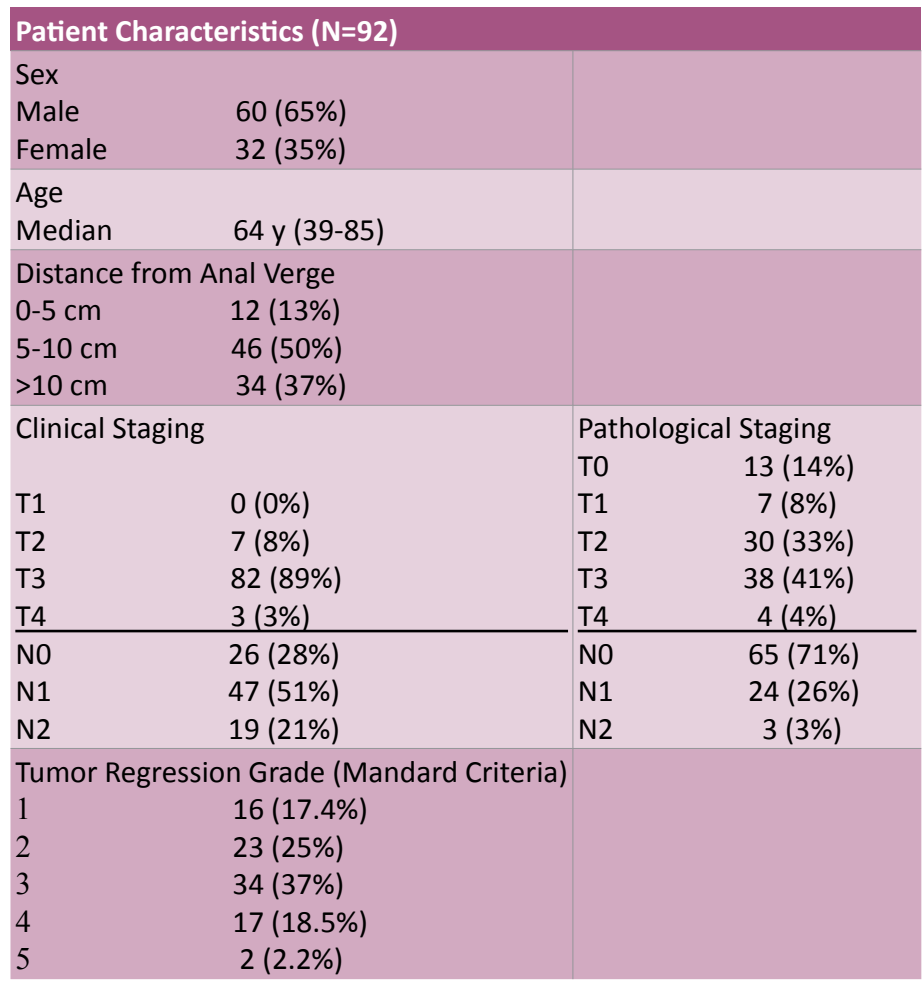


chemotherapy, including OXA in 34 patients (38\%) (Table 2).

The descriptive statistics for these selected 92 patients (two year after LARC with absence of relapse) were; a median follow-up of all the series of 76 months (35-135 months), with 8/92 relapses (two local relapses, four distant relapses and two both local and distant relapses (Free-relapse rate: 82/92: 89.1\%). Five and 10 -year overall survival of these 92 patients were $96.5 \%$ (Cl95\%: 92.58\%-100\%) and 90.0 (CI95\%: 83.06\%-98.74\%), respectively.

Wexner test median values did not change significantly between OXA-treated and untreated patients. The median (IQR) for the group treated without adjuvant OXA was 6.0 (1.0-11.25), and of $5.0(1.0-10.0)$ for the group receiving adjuvant OXA $(p=0.450)$ (Figure 1).

\section{Discussion}

Despite the numerous studies testing chemotherapy-based adjuvant treatment in LARC, the contradictory results from these studies has caused significant controversy on this approach after CRT in LARC. However, our findings support the use of this drug in adjuvant setting, due to the absence of deleterious effects over sphincter anal function. Even more, some sensorial deleterious effects have been attributed to sequential neoadjuvant (nonbased in oxaliplatin) and surgical approach and not to the adjuvant management [10].

The Food and Drug Administration (FDA) indicates that more than $70 \%$ of the patients receiving oxaliplatin are affected by some degree of sensory neuropathy [15]. Most importantly, neurotoxicity and not tumor progression is frequently the cause of treatment discontinuation. There are two patterns of neuropathy: (a) an acute cold-aggravated but transient condition and (b) a more chronic form that appears after multiple exposures to the drug. The chronic form of neuropathy often improves following drug cessation, although it does not fully disappear.

It is a very important question as poor bowel function has adverse consequences on work, social interactions, physical activities and global quality of life. We know that chemoradiation adversely affects function. So, the question whether oxaliplatin with its attendant neurotoxicity adds further problems is indeed an important one-with relevance to patients and everyday practice. Long-term anorectal symptoms seems to correlate more with a combination of changes in anorectal biomechanical properties of the neorectum and rectal mucosal injury. Some data suggests that RT-induced long-term anorectal dysfunction is multifactorial caused by fibrosis of the internal anal sphincter combined with increased rectal sensitivity and reduced rectal functional capacity. Low anterior resection syndrome seems to

Table 2 Adjuvant chemotherapy.

\begin{tabular}{|c|c|c|}
\hline & $\mathbf{N}=\mathbf{9 2}$ patients & \\
\hline FUFA & 17 & $18 \%$ \\
\hline XELOX & 33 & $37 \%$ \\
\hline XEL & 23 & $25 \%$ \\
\hline FOLFOX & 1 & $1 \%$ \\
\hline No QT & 18 & $19 \%$ \\
\hline
\end{tabular}

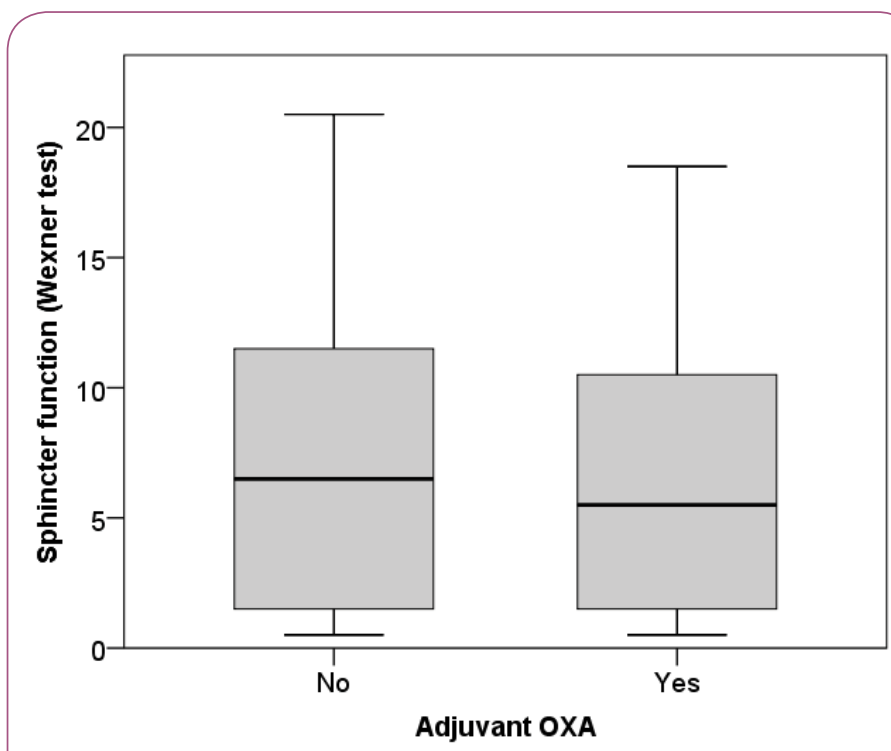

Figure 1 Box-plots for Wexner test results in patients with and without adjuvant OXA.

be caused by physiological changes due to neural damage more than structural changes in the ano-neorectum [16].

We know that pelvic radiotherapy causes hyposensitivity to mechanical and thermal stimuli in the neorectum of patients receiving chemoradiation (CRT) prior to TME, possibly due to impaired afferent nerve function. The combination of surgery and CRT gives rise to much worse symptoms.

Although the Wexner's test is one of the most widely used, it has several disadvantages [17]. Firstly, it does not take account faecal urgency, which can be present without faecal incontinence. Secondly, other scenarios as the need for wearing a diaper should be regarded as equally important as the occurrence of incontinence. The use of a diaper should not be construed as a measure of the severity of faecal incontinence, but it rather reflects the degree of impairment in terms of quality of life and autonomy of the patient. It has to be remarked that the use of diapers is often related to coexistent urinary leakage. Finally, some other factors such as the use of oral antidiarrheal drugs must be assessed pre and postoperatively. Accordingly, other factors that quantify sphincter function such as anal manometry would be important to consider. However, these kind of measurements may cause some reticence in patients, risking their participation in these studies.

By the other side, an internationally validated patient reported outcome measure (PROM), the Low Anterior Resection Syndrome (LARS) score [18], is now available to measure these symptoms. It has five questions: incontinence of flatus; incontinence of liquid stool; frequency; clustering; and, urgency. Each item is individually weighted and a summative score is derived (range 0-42). This scoring tool classifies patients into three severity groups: no LARS (0-20 units), minor LARS (21-29 units) and major LARS (30-42 units). It would be useful to have this measure as well as the Wexner. 
Our present study still presents some limitations. Firstly, the retrospective nature of our study requires the validation of our results in a prospective and larger study. Secondly, 92 patients are simply too small a number to have robust conclusions. Indeed, only 34 patients received oxaliplatin. Of these only $50 \%$ of the cases were located in the medium rectum $(5-10 \mathrm{~cm}$ from anal margin), and only the Wexner score to test sphincter function. Third, although no significant differences were observed after OXA treatment, the decision of applying adjuvant treatment might be influenced by the recovery after surgery. However, our study did not show evidence of this influence or that it affected our conclusions. And finally, the absence of baseline sphincter function evaluation (prior to treatment) make we must assume that the 2 population had similar baseline sphincter function.

Nevertheless, to the best of our knowledge, this is the first study on the effects of OXA in anal function after a sequential approach in LARC. Due to the retrospective nature of our study and different adjuvant treatments administered in this study, additional prospective confirmative studies are required to support our findings.

\section{Acknowledgements}

This study has been supported by a grant of "Mutua Madrileña Fundation". 


\section{References}

1 Gaertner WB, Kwaan MR, Madoff RD, Melton GB (2015) Rectal cancer: An evidence-based update for primary care providers. World J Gastroenterol 21: 7659-7671.

2 Siegel RL, Miller KD, Jemal A (2015) Cancer statistics. CA Cancer J Clin 65: 5-29.

3 Kockerling F, Reymond MA, Altendorf-Hofmann A (1998) Influence of surgery on metachronous distant metastases and survival in rectal cancer. J Clin Oncol 16: 324-329.

4 Nelson H, Petrelli N, Carlin A (2001) National Cancer Institute Expert Panel. Guidelines 2000 for colon and rectal cancer surgery. J Nat Cancer Inst 93: 583-596.

5 Julien LA, Thorson AG (2010) Current neoadjuvant strategies in rectal cancer. J Surg Oncol 101: 321-326.

6 Raymond E, Faivre S, Woynarowski JM, Chaney SG (1998) Oxaliplatin: mechanism of action and antineoplastic activity. Semin Oncol 25 4-12.

7 De Gramont A, Figer A, Seymour M (2000) Leucovorin and fluorouracil with or without oxaliplatin as first-line treatment in advanced colorectal cancer. J Clin Oncol 18: 2938-2947.

8 Van Cutsem E, Cervantes A (2014) Metastatic colorectal cancer: ESMO Clinical Practice Guidelines for diagnosis, treatment and follow-up. Ann Oncol.

9 Bujko K, Glimelius B, Valentini V (2015) Postoperative chemotherapy in patients with rectal cancer receiving preoperative radio(chemo) therapy: A meta-analysis of Randomized trials comparing surgery \pm a fluoropyrimidine and surgery + a fluoropyrimidine \pm oxaliplatin. Eur J Surg Oncol 41: 713-723.
10 Mols F, Beijers T, Lemmens V (2013) Chemotherapy-induced neuropathy and its association with quality of life among 2- to 11year colorectal cancer survivors: results from the population-based PROFILES registry. J Clin Oncol 20: 2699-2707.

11 Carozzi VA, Canta A, Chiorazzi A (2015) Chemotherapy-induced peripheral neuropathy: What do we know about mechanisms? Neurosci Lett 596: 90-107.

12 Cersosimo RJ (2005) Oxaliplatin-associated neuropathy: a review. Ann Pharmacother 39: 128-135.

13 Jorge JMN, Wexner SD (1993) Etiology and management of fecal incontinence. Dis Colon Rectum 36: 77-97.

14 Mandard AM, Dalibard F, Mandard JC, Marnay J, Henry-Amar M, et al (1994) Pathologic assessment of tumor regression after preoperative chemoradiotherapy of esophageal carcicoma. Clinicopathologic correlations. Cancer 73: 2680-2686.

15 Gemlin E, Gamelin L, Bossi L (2002) Clinical aspects and molecular basis of oxaliplatin neurotoxicity: current management and development of preventive measures. Semin Oncol 5: 21-33.

16 Bregendahl S, Emmertsen KJ, Fassov J, Krogh K, Zhao J, et al. (2013) Neorectal hyposensitivity after neoadjuvant therapy for rectal cancer. Radiother Oncol 108: 331-336.

17 Tina C, Katrine J (2015) Emmertsen and Soren Laurberg. What are the Best Questionnaires to Capture Anorectal Function After Surgery in Rectal Cancer?. Curr Colorectal Cancer Rep 11: 37-43.

18 Emmertsen KJ, Laurberg S (2012) Low anterior resection syndrome score: development and validation of a symptom-based scoring system for bowel dysfunction after low anterior resection for rectal cancer. Ann Surg 5: 922-928. 